\title{
Scores of asthma and asthma severity reveal new regions of linkage in EGEA study families
}

\author{
E. Bouzigon*,\#, V. Siroux ${ }^{\top}$, M-H. Dizier ${ }^{+, \S}$, A. Lemainque ${ }^{f}$, C. Pison**,\#\#, M. Lathrop ${ }^{f}$, \\ F. Kauffmann ${ }^{\S, \# \#, ~ F . ~ D e m e n a i s * ~ a n d ~ I . ~ P i n ~}{ }^{\#}$
}

ABSTRACT: There is ongoing debate as to how asthma should be defined in order to forward understanding of the underlying mechanisms. The aim of the present study was to build quantitative scores of asthma and asthma severity and to assess whether refinement of disease phenotypes can facilitate the identification of chromosomal regions harbouring susceptibility genes.

A genome-wide linkage scan was conducted in 110 families with at least two asthmatic siblings $(n=508)$ from the French Epidemiological study on the Genetics and Environment of Asthma, bronchial hyperresponsiveness and atopy (EGEA). The phenotypes studied were an asthma severity score (assessed among asthmatics by combining clinical data and treatment), forced expiratory volume in one second $\left(F E V_{1}\right)$ and an asthma score (including both asthmatics and nonasthmatics and representing the whole disease spectrum).

This analysis showed genome-wide suggestive evidence of linkage of the asthma score to 18 11, a novel region undetected in a previous screen of dichotomous asthma. There was potential linkage of 2 p23 to asthma severity score and of three regions (1p36, $2 q 36$ and $6 q 14)$ to FEV1. Moreover, FEV1 appeared to have no genetic determinant in common with asthma severity and asthma scores.

Asthma and asthma severity quantitative scores revealed new regions of linkage and thus provide support for considering these phenotypes in future genetic studies.

KEYWORDS: Asthma, asthma severity, genetic, heterogeneity, linkage analysis, lung function

$\mathbf{T}$ he general consensus now emerging is that asthma is unlikely to be a single disease, but rather a collection of different phenotypes, as recently pointed out in the Lancet [1]. These phenotypes may be defined from various criteria, including clinical and physiological characteristics (e.g. severity, resistance to treatment, chronic airflow restriction, etc.) [2]. One strategy for determining whether these phenotypes represent different manifestations of a common underlying pathological process or are distinct disease entities is to refine phenotypic characterisation and search for determinants of these phenotypes. Indeed, improving phenotype definition can increase the power to detect the underlying genetic mechanisms and, conversely, genetic analysis can provide support for new phenotype definitions and new phenotypic entities.

Regarding the asthma phenotype, it has recently been shown that use of quantitative scores based on symptoms of asthma [3], rather than simple dichotomous definitions, can improve the identification of risk factors by reducing misclassification of disease status. Definition of asthma severity depends upon its primary purpose. The 2002 and 2004 Global Initiative for Asthma (GINA) guidelines, established to improve asthma management, combine current symptoms, respiratory function and treatment [4] However, it is now recognised that asthma severity is heterogeneous, being represented by different phenotypes that may result from different risk factors [2]. Low lung function is a marker of asthma severity. It antedates the occurrence of persistent wheeze, tracks over the life span, and predicts the severity of asthma in adults in the general population [5] and exacerbations in clinical settings [6]. Asthma occurrence, asthma severity and forced expiratory volume in one second (FEV1) may result from common determinants,
AFFILIATIONS

*INSERM, U794,

\#Université d'Evry, and,

${ }^{\dagger}$ National Genotyping Centre, Evry,

"INSERM, U823 and,

**INSERM, U884, Grenoble, and

+INSERM, U535,

\#\#INSERM, U780, and

sUniversité Paris-Sud, IFR69

Villejuif, France.

CORRESPONDENCE

E. Bouzigon

INSERM U794

Tour Evry 2

523 Place des Terrasses de l'Agora 91034 Evry

France

Fax: 33160873848

E-mail: bouzigon@evry.inserm.tr

Received:

December 122006

Accepted after revision:

April 182007

\section{SUPPORT STATEMENT}

This study was supported, in part, by a National Institute for Health and

Medical Research (INSERM)-

Ministry of Research (Paris, France)

Cohorts and Collections grant

(4CH06G), the EU Framework

programme (Brussels, Belgium) for research (contract FOOD-CT-2004506378), GA²LEN project (Global Allergy and Asthma European Network project; Ghent, Belgium) and the French National Research Agency (Paris, France; ANR 05-SEST-02002/05-9-97). Support was also provided by INSERM, the University of Evry and Génopole (both Evry, France), the Foundation for Medical Research (Paris, France) and AGIR à dom (Meylan, France). 
but also from specific ones. Analysing these phenotypes separately rather than combining them in a single index makes it possible to discriminate between these hypotheses.

Evidence for familial resemblance of asthma severity and the absence of a significant relationship between asthma severity in probands and the occurrence of disease in relatives have suggested that specific familial factors, possibly genetic, are involved in asthma severity [7]. Many genome-wide scans, including the Epidemiological study on the Genetics and Environment of Asthma, bronchial hyperresponsiveness and atopy (EGEA), have reported chromosomal regions linked to asthma, based on classical dichotomous definitions [8]. Although candidate genes for severe asthma have been investigated [9], limited information is available regarding the localisation of potential new genes. To the present authors' knowledge, no genome scan has yet been conducted for categorical phenotypes of asthma and asthma severity.

The EGEA study, with detailed data permitting the construction of scores for asthma and asthma severity in family members, and the availability of linkage analysis methods for ordered categorical traits make it possible to conduct a genome-wide screen for the three following phenotypes: 1) a categorical asthma score, in order to reduce potential asthma misclassification and to investigate the whole spectrum of disease expression; 2) an asthma severity score, in order to investigate variation in disease severity; and 3) a measure of ventilatory function, FEV1, an objective marker of asthma severity. This genome screen permits evaluation of whether the examination of more-refined disease phenotypes can help in the identification of novel regions of linkage. It also permits assessment of whether the three phenotypes under study are more likely to share genes or depend upon different genetic factors.

\section{METHODS AND MATERIALS}

The protocol for the EGEA data collection has been described elsewhere $[7,8,10]$. Subjects answered a detailed questionnaire regarding respiratory symptoms and treatment based on international standardised questionnaires. FEV1 was measured according to American Thoracic Society recommendations.

Among the whole EGEA family sample ascertained through one asthmatic proband, 119 nuclear families including at least two asthmatic siblings were selected for the present study. Asthma was defined using the same criteria as in previous EGEA linkage scans [8], associating report of asthma attacks or attacks of breathlessness with wheezing ever and either airway hyperresponsiveness or reversible obstruction or hospitalisation for asthma or asthma treatment.

\section{Phenotypes analysed}

Asthma severity

Based on extensively documented phenotypes in EGEA, two measures of asthma severity were considered in the present study: 1) asthma severity score; and 2) FEV1.

\section{Asthma severity score}

The asthma severity score, ranging $1-4$, was built in asthmatic siblings in two steps, following the concept of combining treatment and symptoms according to the 2002 and 2004 GINA guidelines [4]. In step 1, the sum was computed, in respect of the last 12 months, of the following clinical items: frequency of asthma attacks ( $<1$ attack per month $(0),<1$ attack per week (1), $<1$ attack per day (2) and $\geqslant 1$ attack per day (3)); symptoms between asthma attacks (none (0), wheezing (1), wheezing and dyspnoea (2), and activities limited by dyspnoea (3)); and hospitalisation for asthma $(\mathrm{no}(0) / \mathrm{yes}(1))$. This sum of clinical items was grouped into four classes: $0,1,2$ and $\geqslant 3$. In step 2, the four-class variable built in step 1 was combined with the type of anti-asthmatic treatment during the past 12 months, as reported in the EGEA questionnaire (no treatment, treatment without inhaled steroids and treatment with inhaled steroids), resulting in an asthma severity score of $1,2,3$ or 4 , as shown in table 1 .

\section{Forced expiratory volume in one second}

Ventilatory function was assessed by means of the FEV1 measured before bronchodilator use, according to a previously described protocol [10], and expressed as a percentage of the predicted value based on age, height and sex [8].

\section{Asthma score}

An asthma score was built to represent the whole disease spectrum, encompassing nonasthmatics and asthmatics with varying asthma expression. This score ranged $0-4$; a score of 0 was assigned to nonasthmatics and the scores of 1-4 represented the four classes of asthma severity defined above.

\section{Genotypes}

Genotyping of 396 microsatellites, with a mean spacing of 10 centimorgans (cM), was performed in EGEA families with at least two siblings with available DNA, following a protocol described elsewhere [8]. After rigorous genotype quality control, the final sample for the present analysis included 110 families with at least two asthmatic siblings (508 subjects), comprising 218 genotyped parents (99\% of all parents) and 288 genotyped siblings.

\section{Linkage analysis}

Linkage analyses of the two ordered categorical phenotypes (asthma severity score and asthma score) were performed using the maximum-likelihood-binomial method [11, 12] implemented in MLB-GENEHUNTER [11]. This is a unique linkage analysis method for categorical traits. The principle of

\begin{tabular}{|c|c|c|c|c|}
\hline \multirow{2}{*}{$\begin{array}{l}\text { Sum of clinical } \\
\text { items }\end{array}$} & \multirow[t]{2}{*}{ Subjects $\mathrm{n}$} & \multirow[t]{2}{*}{ No treatment } & \multicolumn{2}{|c|}{ Treatment } \\
\hline & & & Without ICS & With ICS \\
\hline \multicolumn{2}{|l|}{ Subjects $n$} & 70 & 64 & 79 \\
\hline 0 & 124 & $1(48)$ & $1(42)$ & $2(34)$ \\
\hline 1 & 36 & $1(9)$ & $2(9)$ & $3(18)$ \\
\hline 2 & 33 & $2(8)$ & $3(8)$ & $4(17)$ \\
\hline$\geqslant 3$ & 20 & $3(5)$ & $4(5)$ & $4(10)$ \\
\hline \multicolumn{5}{|c|}{$\begin{array}{l}\text { Data are presented as score (n), unless otherwise stated. The clinical items and } \\
\text { treatment were each defined in respect of the last } 12 \text { months. The clinical items } \\
\text { included the following: frequency of asthma attacks; symptoms between } \\
\text { attacks; and hospitalisation. ICS: inhaled corticosteroid. }{ }^{\#}: 1-4 \text { (based on } \\
\text { combining the sum of clinical items (step 1) and type of treatment (step 2) in } \\
213 \text { genotyped asthmatic siblings). }\end{array}$} \\
\hline
\end{tabular}


this approach is to introduce a latent (unobserved) binary variable $(Y=\{0 ; 1\})$ that captures the linkage information between the observed categorical phenotype and the marker. For each phenotypic category, the latent variable $\mathrm{Y}$ can take a value of 1 (affected), with probability $p_{i}$, or a value of 0 (unaffected), with probability $1-\mathrm{p}_{\mathrm{i}}$. Linkage is then investigated for all possible sets of $Y$ values within sibships weighted by their probabilities $\left(2^{\mathrm{s}}\right.$ sets for a sibship of size $\left.\mathrm{s}\right)$. Choice of these probabilities is guided by the hypothesis under test. The idea is that the probability of $Y=1$ increases as the severity of asthma increases. Different sets of probabilities were used to model the correspondence between the observed category and the unobserved latent variable. When analysing the asthma severity score, the four probabilities assigned to the four classes (1, 2, 3 and 4) were chosen in order to provide maximal power for detecting genes controlling the mildest (classes 1 and 2, having low probabilities of 0.05 and 0.25 for the latent variable $Y$ to be 1 ) or the most severe forms of asthma (classes 3 and 4, having high probabilities of 0.75 and 0.95 for $Y$ to be 1). When analysing the asthma score, the probabilities modelling the correspondence between this score and the latent variable were chosen to investigate a continuum of disease expression and thus ranged from 0 in nonasthmatics to 1.0 in asthmatics belonging to severity class 4 , intermediate probabilities of $0.25,0.45$ and 0.75 being assigned to classes 1, 2 and 3, respectively. The likelihood of the observations is expressed using a binomial distribution of parental marker alleles among offspring according to the value of the binary latent variable and depends upon only one parameter, $\alpha$, the probability that siblings with $Y=1$ receive one of the two marker alleles with the disease allele from his/her heterozygous parent, $1-\alpha$ being the corresponding probability among siblings with $Y=0$. The null hypothesis of no linkage $(\alpha=0.5)$ against the alternative hypothesis of linkage $(\alpha>0.5)$ was tested using a likelihood-ratio test. It is of note that contribution to linkage information comes from sets of siblings having the same value of the latent variable (concordant siblings for $Y=1$ or $Y=0$ ) and from sets of siblings having opposite values of the latent variable (discordant siblings).

Multipoint linkage analysis of the continuous phenotype (FEV1 $\%$ pred) was conducted using the variance-components method [13] implemented in MERLIN [14]. The variancecomponents method separates the total variation of a trait into genetic and environmental components and evaluates linkage by comparing a model incorporating both a genetic additive variance at a putative quantitative trait locus and a polygenic component with a purely polygenic model (quantitative trait locus variance set to zero) using a likelihood-ratio test. Since the variance-components method is known to be sensitive to departure from normality assumptions of the trait distributions, a probit transformation was applied prior to linkage analysis to $\mathrm{FEV} 1$ (\% pred) in order to normalise its distribution.

For either method, minus twice the natural logarithm of this likelihood ratio is assumed to follow a one-sided Chi-squared distribution with one degree of freedom. This Chi-squared distribution divided by $2 \ln 10$ can be considered the logarithm of odds (LOD) score.

\section{Correlations among genome-wide LOD scores}

In order to assess whether or not the asthma score, asthma severity score and FEV1 (\% pred) might share common genetic determinants, the correlation matrix was computed among the standardised genome-wide LOD scores obtained at 378 marker positions for these three phenotypes.

\section{RESULTS \\ Sample characteristics}

The relationship between the asthma severity score and the sum of clinical items is presented in table 1. Taking treatment into account changed the scoring based on clinical items for 91 $(42.7 \%)$ siblings, three-quarters of these siblings being reclassified as more severe and a quarter as less severe compared with step 1 when treatment was ignored.

The 288 genotyped siblings of the 110 nuclear families considered in the present analysis had a mean age of 15 yrs (range $3-43$ yrs) and comprised $82 \%$ asthmatics. The principal characteristics of the asthmatic and nonasthmatic siblings are presented in table 2 .

Regarding the asthma score, which included asthmatic and nonasthmatic siblings, $18 \%$ of these siblings belonged to class 0 , $38 \%$ to class $1,19 \%$ to class 2 , and $12 \%$ each to classes 3 and 4 .

\section{Genome-wide screen}

The genome-wide linkage test results for asthma score, asthma severity score and FEV1 (\% pred) are shown in figure 1 and table 3.

The most significant evidence for linkage was found on chromosome 18p11, for asthma score, where the maximum LOD score reached 2.40 at D18S53, with a p-value of 0.0004 , lower than the critical threshold of $7 \times 10^{-4}$ for genome-wide suggestive evidence of linkage [15]. There was modest evidence of linkage to asthma score $(\mathrm{p}<0.01)$ in two other regions: 5q15 (LOD 1.22 at D5S428; $\mathrm{p}=0.009$ ) and 14q13 (LOD 1.20 at D14S70; $\mathrm{p}=0.009)$.

In respect of the asthma severity score, a maximum LOD score of $1.80(p=0.002)$ was obtained on chromosome $2 \mathrm{p} 23$, at marker D2S165. Additional potential linkage was observed in the 7q36 region (LOD 1.00 at D7S2465; p=0.01).

Three regions were found to be linked to FEV1 (\% pred) at $\mathrm{p} \leqslant 0.005$ : $1 \mathrm{p} 36$ (LOD 1.52 at D1S468; $\mathrm{p}=0.004), 2 \mathrm{q} 36$ (LOD 1.59 at D2S126; $\mathrm{p}=0.003)$ and 6q14 (LOD 1.64 at D6S460; $\mathrm{p}=0.003)$ There were two additional linkage signals at the $1 \%$ significance level: 4p14 (LOD 1.21 at D4S405; p=0.009) and 7p22 (LOD 1.19 at D7S531; $\mathrm{p}=0.01$ ).

\section{Correlations among genome-wide LOD scores}

Estimation of the correlations among genome-wide LOD scores for the three phenotypes showed nonsignificant correlations that were close to zero between LODs for FEV1 (\% pred) and LODs for asthma score $(\mathrm{r}=-0.03)$ and asthma severity score $(r=-0.07)$, respectively. Thus FEV1 (\% pred) appears to have no genetic factor in common with the asthma score and asthma severity score. There was a significant positive correlation, although not very high, between LODs for the asthma score and asthma severity score $(r=0.13 ; p=0.01)$, suggesting that these phenotypes may share a few genetic factors.

\section{DISCUSSION}

There is ongoing debate as to how asthma should be defined in order to progress understanding of the underlying mechanisms 


\begin{tabular}{|c|c|c|}
\hline TABLE 2 & \multicolumn{2}{|c|}{$\begin{array}{l}\text { Phenotypic characteristics of genotyped siblings } \\
\text { in the set of } 110 \text { families with at least two } \\
\text { asthmatic siblings }\end{array}$} \\
\hline \multicolumn{2}{|c|}{ Asthmatic siblings $n$} & 237 \\
\hline \multicolumn{2}{|c|}{ Males } & $142(59.7)$ \\
\hline \multicolumn{2}{|l|}{ Age yrs } & $14.7 \pm 7.3$ \\
\hline \multicolumn{2}{|c|}{ FEV $1 \%$ pred } & $95.1 \pm 13.4$ \\
\hline \multicolumn{2}{|c|}{ Age at asthma onset yrs } & $5.9 \pm 5.5$ \\
\hline \multicolumn{3}{|c|}{ Frequency of asthma attack ${ }^{\#}$} \\
\hline \multicolumn{2}{|c|}{ None } & $43(19.0)$ \\
\hline \multicolumn{2}{|c|}{$<1$ attack per month } & $104(46.0)$ \\
\hline \multicolumn{2}{|c|}{$\geqslant 1$ attack per month; $<1$ attack per week } & 45 (19.9) \\
\hline \multicolumn{2}{|c|}{$\geqslant 1$ attack per week; $<1$ attack per day } & $27(12.0)$ \\
\hline \multicolumn{2}{|c|}{$\geqslant 1$ attack per day } & $7(3.1)$ \\
\hline \multicolumn{2}{|c|}{ Persistent symptoms between attacks } & $32(15.0)$ \\
\hline \multicolumn{2}{|c|}{ Hospitalisation\# } & $14(6.3)$ \\
\hline \multicolumn{3}{|c|}{ Sum of clinical items } \\
\hline \multicolumn{2}{|c|}{0} & $124(58.2)$ \\
\hline \multicolumn{2}{|l|}{1} & $36(16.9)$ \\
\hline \multicolumn{2}{|l|}{2} & $33(15.5)$ \\
\hline \multicolumn{2}{|l|}{$\geqslant 3$} & $20(9.4)$ \\
\hline \multicolumn{3}{|l|}{ Treatment ${ }^{\#}$} \\
\hline \multicolumn{2}{|l|}{ None } & $80(33.7)$ \\
\hline \multicolumn{2}{|c|}{ With ICS } & $86(36.3)$ \\
\hline \multicolumn{2}{|c|}{ Without ICS } & $71(30.0)$ \\
\hline \multicolumn{3}{|c|}{ Asthma severity score } \\
\hline \multicolumn{2}{|c|}{1} & $100(46.7)$ \\
\hline \multicolumn{2}{|l|}{2} & $51(23.8)$ \\
\hline \multicolumn{2}{|l|}{3} & $31(14.5)$ \\
\hline \multicolumn{2}{|l|}{4} & $32(14.9)$ \\
\hline \multicolumn{2}{|c|}{ Nonasthmatic siblings $\mathrm{n}$} & 49 \\
\hline \multicolumn{2}{|c|}{ Males } & $26(53.1)$ \\
\hline \multicolumn{2}{|c|}{ Age yrs } & $15.4(10.1)$ \\
\hline \multicolumn{2}{|c|}{ FEV $1 \%$ pred } & $100.7(16.2)$ \\
\hline
\end{tabular}

Data are presented as mean \pm SD or $n(\%)$, unless otherwise stated.FEV 1 : forced expiratory volume in one second; \% pred: \% predicted; ICS: inhaled

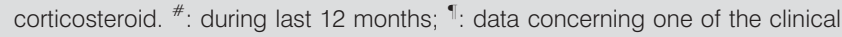
items (persistent symptoms between attacks) was available for 213 siblings, and thus the sum of clinical items was determined in 213 out of the 237 genotyped asthmatic siblings.

involved [1]. To date, genetic studies have used dichotomous definitions, often established from questionnaires. The present genome-wide scan is the first to consider varying levels of asthma expression (from unaffected to severely affected). The asthma spectrum phenotype, encompassing nonasthmatics and various classes of asthma, showed genome-wide suggestive evidence for linkage to the $18 \mathrm{p} 11$ region. The asthma severity score, combining clinical items and treatment, showed linkage to the 2 p23 region, whereas other linkage signals were detected for FEV1 on chromosomes 1p36, 2q36 and 6q14. None of the regions detected for any of these three phenotypes was revealed for the other two. Moreover, estimation of the correlations among genome-wide LOD scores showed no evidence for any common genetic determinant between FEV1 (\% pred) and either the asthma score or asthma severity score, whereas there was suggestion of the sharing of a few genetic factors by these two scores. These potential common genetic determinants are likely to have weak effects since no region common to the latter two phenotypes was detected at the $1 \%$ significance level.

\section{Phenotype definition and linkage analysis outcomes}

The strongest evidence for linkage was found for the asthma score, on 18p11. Previous analysis of asthma in the same EGEA sample, using a dichotomous definition, had revealed a single linkage signal in the $1 \mathrm{p} 31$ region $(\mathrm{p}=0.005)$ [8]. Further analysis led to increased evidence for linkage to this region when affection status was defined by the presence of both asthma and allergic rhinitis, and showed that 1 p31 is likely to contain a gene specific to this comorbid condition [16]. Conversely, the $18 \mathrm{p} 11$ region, revealed by the present analysis of categorical asthma score, was not detected in a previous analysis of dichotomous asthma and seven other asthma-related phenotypes [8]. This shows that considering a more refined phenotype, including various classes of asthma expression, can lead to the detection of new genes influencing variation in disease expression. Finding new regions by considering a categorical definition for asthma raises several hypotheses. First, these results may be due to a reduction in asthma misclassification. Whereas asthmatic probands were defined using a very strict procedure and recruited in chest clinics [10], the definition of asthma in siblings was less specific, although attention was paid to items such as bronchial hyperresponsiveness, treatment or hospitalisation in the confirmation of asthma. In this context, a categorical variable may decrease such potential misclassification bias. Indeed, a second possibility is that asthma is not a dichotomous phenotype, but, as with airflow limitation, may occur on a somewhat continuous scale. A similar approach of building continuous scores for asthma was proposed by PEKKANEN et al. [3], and was shown to increase the power of detecting risk factors for disease. The present score for asthma was constructed upon the asthma severity score, itself defined from clinical symptoms and treatment, but other types of continuous score may be built. Interestingly, PEKKANEN et al. [3] found that a simple sum of positive answers to eight questions regarding clinical symptoms and asthma medication, i.e. based on the same principles as the present grading of asthma severity, was as effective at detecting risk factors as a score computed from a more sophisticated principal component analysis of 12 questions regarding asthma symptoms. Thus, the present results and those of PEKKANEN et al. [3] provide strong support for the usefulness of examining quantitative scores of asthma in aetiological research.

It was decided not to include FEV1 in the severity score, despite the fact that this physiological measure may be helpful in the assessment of asthma severity in clinical settings, since the present aim was to investigate whether ventilatory function and the asthma severity score result from common or distinct genetic determinants. The present results show that FEV1 (\% pred) and the asthma severity score do not share any genetic determinant. This is in agreement with other phenotypic studies, which have shown that FEV1 does not systematically strongly correlate with disease symptoms [2, 17]. Moreover, none of the published genome-wide screens conducted for lung function in asthmatic families reported linkage to the 2 p23 region, detected here for the asthma severity score [18-20]. 


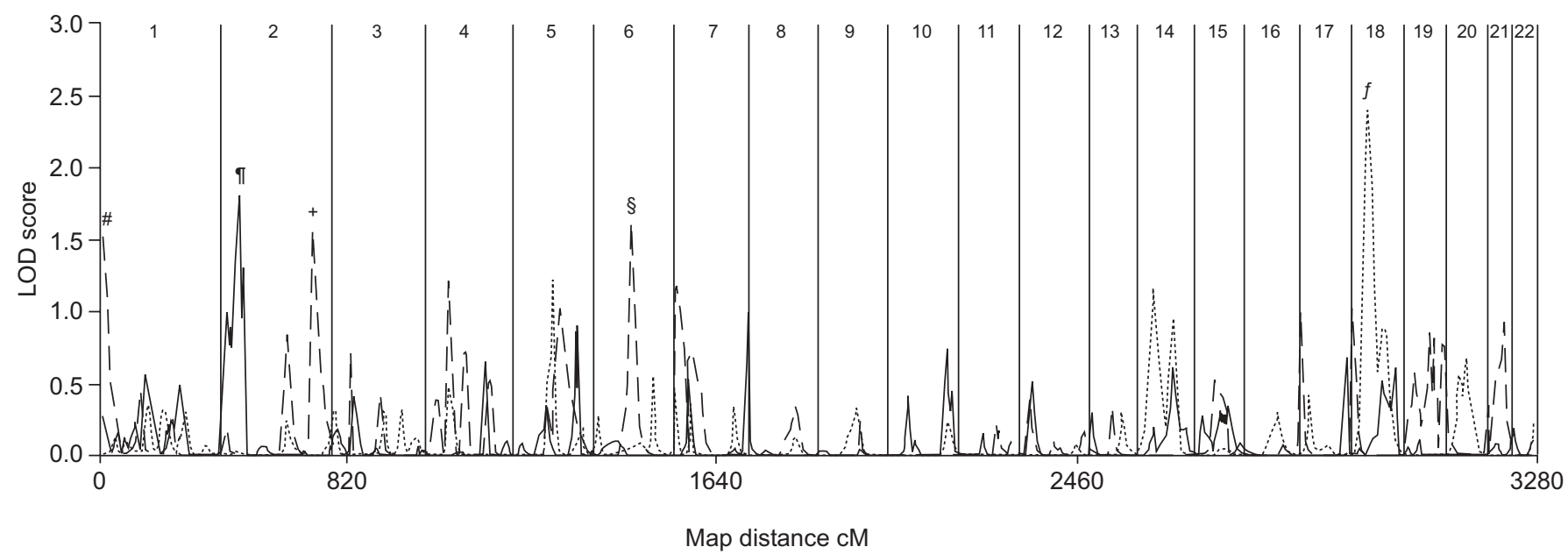

FIGURE 1. Multipoint results of the genome-wide linkage scan for asthma score (-----), asthma severity score ( $\longrightarrow$ ) and forced expiratory volume in one second percentage of the predicted value (-- - ) conducted in 110 Epidemiological study on the Genetics and Environment of Asthma, bronchial hyperresponsiveness and atopy (EGEA) families. The microsatellite markers were as follows: ${ }^{\#}:$ D1S468 $(p=0.004) ;{ }^{\circ}:$ D2S165 $(p=0.002) ;{ }^{+}:$D2S126 $(p=0.003) ;{ }^{\$}:$ D6S460 ( $\left.p=0.003\right) ;{ }^{f}:$ D18S53 ( $\left.p=0.0004\right)$. Numbers above the $\mathrm{x}$-axis represent chromosome number. cM: centimorgan.

Taken together, these results indicate that FEV1 (\% pred) is more likely to be controlled by specific genetic determinants. Thus, in the search for genetic determinants of asthma severity, it may be worthwhile to consider lung function separately from clinical symptoms and treatment. It should also be noted that the 2p23 region found to be linked to asthma severity score was not revealed by a previous scan of dichotomous asthma and seven asthma-associated phenotypes [8], and thus appears as a new and specific region of asthma severity in the present sample. Among the three linkage signals revealed for FEV1, only the 6q14 region was detected by the previous scan of the whole EGEA sample with at least one asthmatic proband, and even more significantly $(\mathrm{p}=0.001)$, suggesting that genes in this region may rather control physiological variation of this phenotype.

\section{Replication of linkage results}

It has often been advocated that replication of linkage results across studies provides support for the genuine involvement of linkage regions. An exhaustive compilation of the linkage results of the published genome scans of asthma and asthmaassociated phenotypes performed to date, in 16 different

TABLE 3 Chromosomal regions showing significant $(p \leqslant 0.01)$ multipoint logarithm of odds $(L O D)$ scores for at least one phenotype $^{\#}$

\begin{tabular}{|c|c|c|c|c|c|c|c|}
\hline \multirow[t]{2}{*}{ Marker } & \multirow[t]{2}{*}{ Position ${ }^{\bullet} \mathrm{cM}$} & \multicolumn{2}{|c|}{ Asthma score } & \multicolumn{2}{|c|}{ Asthma severity } & \multicolumn{2}{|c|}{ FEV1 (\% pred) } \\
\hline & & LOD & $p$-value & LOD & p-value & LOD & p-value \\
\hline D1S468 & 4.20 & & & & & 1.52 & 0.004 \\
\hline D1S214 & 14.00 & & & & & 1.16 & 0.01 \\
\hline D2S305 & 38.90 & & & 1.46 & 0.005 & & \\
\hline D2S165 & 47.40 & & & 1.80 & 0.002 & & \\
\hline D2S126 & 221.10 & & & & & 1.59 & 0.003 \\
\hline D4S405 & 56.95 & & & & & 1.21 & 0.009 \\
\hline D5S428 & 95.40 & 1.22 & 0.009 & & & & \\
\hline D6S460 & 89.80 & & & & & 1.64 & 0.003 \\
\hline D7S531 & 5.28 & & & & & 1.19 & 0.01 \\
\hline D7S517 & 7.14 & & & & & 1.14 & 0.01 \\
\hline D7S2465 & 180.4 & & & 1.00 & 0.01 & & \\
\hline D14S70 & 40.11 & 1.20 & 0.009 & & & & \\
\hline D18S464 & 31.17 & 2.06 & 0.001 & & & & \\
\hline D18S53 & 41.24 & 2.40 & 0.0004 & & & & \\
\hline D18S478 & 52.86 & 1.37 & 0.006 & & & & \\
\hline
\end{tabular}

\# : in 110 Epidemiological study on the Genetics and Environment of Asthma, bronchial hyperresponsiveness and atopy (EGEA) families; ${ }^{\bullet}$ of linkage peak, based on Marshfield map. cM; centimorgan; FEV1: forced expiratory volume in one second; \% pred: \% predicted. 
populations (not including the EGEA study), was carried out. All previously reported linkage peaks $(\mathrm{p} \leqslant 0.01)$ spanning a 20 $\mathrm{cM}$ region on either side of each of the present five main linkage peaks were considered. Among the 16 asthma genome scans, the 18p11 region, detected for asthma score, was reported once in Australian twins for asthma analysed as a binary trait [18]. The linkage of the asthma severity score to 2p23 was detected for eosinophils in Australian twins [18, 21, 22] and for severe asthma in German data [23]. It should be noted that the latter study examined dichotomous subtypes of asthma, which were defined, using broader categorisation than the present study, from the three following items: asthma attack frequency, receiving asthma treatment or not, and experience or otherwise of at least one overnight hospital stay. Since FEV1 (\% pred) was studied in only four asthma genome screens, linkage scans conducted for lung function phenotypes in families with early-onset chronic obstructive pulmonary disease (COPD) and from the general population were considered [24]. Among the three linkage signals detected for FEV1, 1p36 was reported to be linked to asthma in two scans $[22,25]$ and $6 q 14$ to eosinophils in one scan [26], whereas linkage to $2 q 36$ was observed for atopy phenotypes in two asthma scans $[18,21,22]$ and for lung function phenotypes in asthmatic families [19] and early-onset COPD families [27, 28]. Regarding this latter result, longitudinal studies have shown that asthma is associated with accelerated lung function decline and is a risk factor for COPD [29]. Thus genetic factors on chromosome $2 \mathrm{q}$ might contribute to the common determinants of asthma and COPD.

\section{Limitations and strengths}

A major strength of the present study was the availability of extensively documented phenotypes for all subjects in the EGEA sample, ascertained through asthmatic cases followed in chest clinics. This permitted the study of a wide range of asthma severity, but it is acknowledged that one limitation of the present study is the small number of severe asthmatics, such as those patients included in specific studies of this asthma sub-phenotype [2]. Examination of the families that contributed to linkage to $2 \mathrm{p} 23$ came mainly from siblings affected with mild asthma, as well as from discordant sets of siblings with mild and severe asthma, suggesting that this region may harbour genes underlying mild asthma and possibly protecting from severe asthma.

The assessment of asthma severity remains a difficult issue because of the paucity of knowledge and heterogeneity of this condition, as well as rapidly evolving concepts regarding its measurement. Here, it was decided to combine clinical items and treatment in order to follow the same principle as recommended in the 2002 and 2004 GINA guidelines. Despite the fact that the dose of treatment was not available in the present data, it was possible to assess the use of inhaled corticosteroids during the past 12 months. Inhaled corticosteroids are the main controller medication and differentiate intermittent from persistent asthma in the GINA guidelines. Moreover, the asthma severity classification presently used has already been used to examine the relationship between occupational exposure and asthma severity in the EGEA data [30].

The present authors are aware that the latest GINA recommendations (2006) have evolved and shifted from severity to control. However, since this approach is adapted to clinical practices for the management of individual asthmatic patients, it may not be the best approach for epidemiological studies, aimed at determining risk factors for asthma severity in populations. In cross-sectional surveys, the level of asthma symptoms may not be interpreted in the same way in asthmatic populations with and without controller medication. Taking medication into account may provide useful additional information in populations, as underlined by LIARD et al. [31], who showed that a classification of asthma severity combining treatment with symptoms and FEV1 showed better correlation with emergency visits or hospitalisation for asthma than the classification without treatment. The degree of airway hyperresponsiveness could be also considered an objective marker of asthma severity, but specific criteria for the test in the EGEA led to a large number of missing data in asthmatics, and thus to too few siblings being available for linkage analysis.

Another strength of the present study was the use of a unique method for categorical phenotypes that is very flexible, since the probabilities modelling the correspondence between the observed scores and the underlying latent variable can be chosen to increase the power for testing a given hypothesis. Moreover, this method takes into account, in the same analytical framework, sets of siblings with similar and opposite forms of the disease spectrum (possibly including those that are unaffected), which both contribute to linkage (concordant siblings sharing an excess and discordant siblings sharing a lower proportion of marker alleles that are identical by descent than the distribution expected under the null hypothesis of no linkage) and can thus increase the power for detecting linkage signals.

\section{Conclusion}

In conclusion, the present study shows that the use of a categorical phenotype to represent the whole spectrum of disease expression instead of a simple dichotomous phenotype can increase the power for detecting new genetic regions. It also provides evidence of a specific genetic component involved in asthma severity and that different genetic components underlie the various dimensions of asthma severity represented, on the one hand, by combination of clinical symptoms and treatment and, on the other hand, by forced expiratory volume in one second. These findings have important implication for the consideration of more refined disease phenotypes in future genetic studies of asthma.

\section{ACKNOWLEDGEMENTS}

The members of the Epidemiological study on the Genetics and Environment of Asthma, bronchial hyperresponsiveness and atopy (EGEA) cooperative group are as follows (all France).

Coordination: F. Kauffmann; F. Demenais (genetics); and I. Pin (clinical aspects).

Respiratory epidemiology: V. Siroux (INSERM U578, Grenoble); M. Korobaeff and F. Neukirch (Egea1 (first survey)/INSERM U700, Paris); I. Annesi-Maesano (INSERM U707, Paris); F. Kauffmann, N. Le Moual, R. Nadif and M.P. Oryszczyn (INSERM U780, Villejuif).

Genetics: J. Feingold (INSERM U393, Paris); M.H. Dizier (INSERM U535, Villejuif); E. Bouzigon and F. Demenais 
(INSERM U794, Evry); I. Gut and M. Lathrop (National Genotyping Centre, Evry).

Clinical centres: I. Pin and C. Pison (Grenoble); D. Ecochard (Egea1); F. Gormand and Y. Pacheco (Lyon); D. Charpin (Egea1) and D. Vervloet (Marseille); J. Bousquet (Montpellier); A. Lockhart (Egea1) and R. Matran (Paris Cochin); E. Paty and P. Scheinmann (Paris Necker); A. Grimfeld and J. Just (ParisTrousseau).

Data and quality management: J. Hochez (Egea1/INSERM exU155); N. Le Moual and C. Ravault (INSERM Unit 780, Villejuif); N. Chateigner (INSERM U794); J. Ferran (Grenoble).

\section{REFERENCES}

1 Anon. A plea to abandon asthma as a disease concept. Lancet 2006; 368: 705.

2 Wenzel SE. Asthma: defining of the persistent adult phenotypes. Lancet 2006; 368: 804-813.

3 Pekkanen J, Sunyer J, Anto JM, Burney P. Operational definitions of asthma in studies on its aetiology. Eur Respir J 2005; 26: 28-35.

4 National Heart, Lung, and Blood Institute, Global Strategy for Asthma Management and Prevention. National Institutes of Health Publication No. 02-95-3659. Bethesda, National Institutes of Health, 2004.

5 de Marco R, Marcon A, Jarvis D, et al. Prognostic factors of asthma severity: a 9-year international prospective cohort study. J Allergy Clin Immunol 2006; 117: 1249-1256.

6 Fuhlbrigge AL, Weiss ST, Kuntz KM, Paltiel AD. Forced expiratory volume in 1 second percentage improves the classification of severity among children with asthma. Pediatrics 2006; 118: e347-e355.

7 Pin I, Siroux V, Cans C, et al. Familial resemblance of asthma severity in the EGEA* study. Am J Respir Crit Care Med 2002; 165: 185-189.

8 Bouzigon E, Dizier MH, Krahenbuhl C, et al. Clustering patterns of LOD scores for asthma-related phenotypes revealed by a genome-wide screen in 295 French EGEA families. Hum Mol Genet 2004; 13: 3103-3113.

9 Ober C, Hoffjan S. Asthma genetics 2006: the long and winding road to gene discovery. Genes Immun 2006; 7: 95-100.

10 Kauffmann F, Dizier MH, Pin I, et al. Epidemiological study of the genetics and environment of asthma, bronchial hyperresponsiveness, and atopy: phenotype issues. Am J Respir Crit Care Med 1997; 156: S123-S129.

11 Abel L, Muller-Myhsok B. Robustness and power of the maximum-likelihood-binomial and maximum-likelihoodscore methods, in multipoint linkage analysis of affectedsibship data. Am J Hum Genet 1998; 63: 638-647.

12 Alcais A, Philippi A, Abel L. Genetic model-free linkage analysis using the maximum-likelihood-binomial method for categorical traits. Genet Epidemiol 1999; 17: Suppl. 1, S467-S472.

13 Amos CI. Robust variance-components approach for assessing genetic linkage in pedigrees. Am J Hum Genet 1994; 54: 535-543.

14 Abecasis GR, Cherny SS, Cookson WO, Cardon LR. Merlin - rapid analysis of dense genetic maps using sparse gene flow trees. Nat Genet 2002; 30: 97-101.
15 Lander E, Kruglyak L. Genetic dissection of complex traits: guidelines for interpreting and reporting linkage results. Nat Genet 1995; 11: 241-247.

16 Dizier $\mathrm{MH}$, Bouzigon E, Guilloud-Bataille $\mathrm{M}$, et al. Evidence for a locus in the 1 p31 region specifically linked to the co-morbidity of asthma and allergic rhinitis in the EGEA study. Hum Hered 2007; 63: 162-167.

17 Siroux V, Kauffmann F, Pison C, Pin I. Caractère multidimensionnel de la sévérité de l'asthme dans l'enquête EGEA. [Multidimensional character of asthma severity in the EGEA study.]. Rev Mal Respir 2004; 21: 917-924.

18 Ferreira MA, O'Gorman L, Le Souef $\mathrm{P}$, et al. Robust estimation of experimentwise $\mathrm{P}$ values applied to a genome scan of multiple asthma traits identifies a new region of significant linkage on chromosome 20q13. Am J Hum Genet 2005; 77: 1075-1085.

19 Postma DS, Meyers DA, Jongepier H, Howard TD, Koppelman GH, Bleecker ER. Genomewide screen for pulmonary function in 200 families ascertained for asthma. Am J Respir Crit Care Med 2005; 172: 446-452.

$20 \mathrm{Xu}$ X, Fang Z, Wang B, et al. A genomewide search for quantitative-trait loci underlying asthma. Am J Hum Genet 2001; 69: 1271-1277.

21 Evans DM, Zhu G, Duffy DL, Montgomery GW, Frazer IH, Martin NG. Major quantitative trait locus for eosinophil count is located on chromosome 2q. J Allergy Clin Immunol 2004; 114: 826-830.

22 Ober C, Tsalenko A, Parry R, Cox NJ. A second-generation genomewide screen for asthma-susceptibility alleles in a founder population. Am J Hum Genet 2000; 67: 1154-1162.

23 Altmuller J, Seidel C, Lee YA, et al. Phenotypic and genetic heterogeneity in a genome-wide linkage study of asthma families. BMC Pulm Med 2005; 5: 1.

24 Joost O, Wilk JB, Cupples LA, et al. Genetic loci influencing lung function: a genome-wide scan in the Framingham Study. Am J Respir Crit Care Med 2002; 165: 795-799.

25 Haagerup A, Bjerke T, Schiotz PO, Binderup HG, Dahl R, Kruse TA. Asthma and atopy - a total genome scan for susceptibility genes. Allergy 2002; 57: 680-686.

26 Wjst M, Fischer G, Immervoll $\mathrm{T}$, et al. A genome-wide search for linkage to asthma. German Asthma Genetics Group. Genomics 1999; 58: 1-8.

27 DeMeo DL, Celedon JC, Lange C, et al. Genome-wide linkage of forced mid-expiratory flow in chronic obstructive pulmonary disease. Am J Respir Crit Care Med 2004; 170: 1294-1301.

28 Silverman EK, Palmer LJ, Mosley JD, et al. Genomewide linkage analysis of quantitative spirometric phenotypes in severe early-onset chronic obstructive pulmonary disease. Am J Hum Genet 2002; 70: 1229-1239.

29 Lange P, Parner J, Vestbo J, Schnohr P, Jensen G. A 15-year follow-up study of ventilatory function in adults with asthma. N Engl J Med 1998; 339: 1194-1200.

30 Le Moual N, Siroux V, Pin I, Kauffmann F, Kennedy SM, on behalf of the Epidemiological Study on the Genetics and Environment of Asthma, Asthma severity and exposure to occupational asthmogens. Am J Respir Crit Care Med 2005; 172: 440-445.

31 Liard R, Leynaert B, Zureik M, Beguin FX, Neukirch F. Using global initiative for asthma guidelines to assess asthma severity in populations. Eur Respir J 2000; 16: 615-620. 